
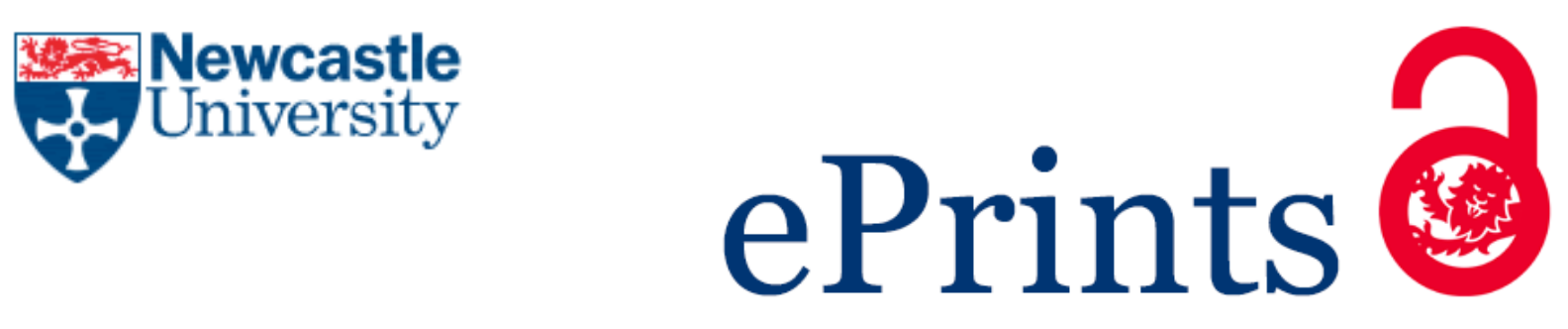

Mayes J, Brown MC, Davies N, Skinner R.

Health promotion and information provision during long-term follow-up for childhood cancer survivors: a service evaluation. Pediatric Hematology and Oncology 2016, 33(6), 359-370.

\title{
Copyright:
}

This is an Accepted Manuscript of an article published by Taylor \& Francis in Pediatric Hematology and Oncology on 30/09/2016, available online: http://dx.doi.org/10.1080/08880018.2016.1225325

Date deposited:

$09 / 12 / 2016$

Embargo release date:

30 September 2017

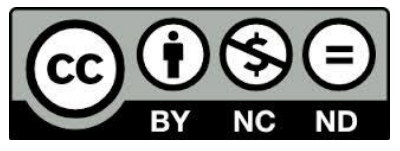

This work is licensed under a

Creative Commons Attribution-NonCommercial-NoDerivatives 4.0 International licence 


\section{Health promotion and information provision during long-term follow up for childhood cancer survivors: a service evaluation}

Jonathan Mayes ${ }^{1}$, Morven C Brown ${ }^{1}$, Nicola Davies ${ }^{2}$, and Roderick Skinner ${ }^{1,2}$

${ }^{1}$ Newcastle University, Newcastle upon Tyne, UK

${ }^{2}$ Great North Children's Hospital, Newcastle upon Tyne, UK

\section{Correspondence to:}

Professor Roderick Skinner, Department of Paediatric and Adolescent Haematology /

Oncology, Great North Children's Hospital, Royal Victoria Infirmary, Newcastle upon Tyne. NE1 4LP. UK.

Tel: 01912336161 Ext 24140 Fax: 01912824726 Email: roderick.skinner@ ncl.ac.uk

Word count abstract: 249

Number of figures: 1

Number of tables: 2

Short running title: Health promotion and information provision for CCS

Keywords: Childhood cancer survivors; health promotion; health education, service evaluation

Abbreviation Table

\begin{tabular}{|r|l|}
\hline CCS & Childhood Cancer Survivors \\
\hline LAE & Late Adverse Effect \\
\hline LTFU & Long-Term Follow-Up \\
\hline
\end{tabular}




\section{Abstract}

Health promotion is an important component of long-term follow-up (LTFU) care for childhood cancer survivors (CCS). However, little information exists about how survivors perceive their own health promotion needs. As part of a service evaluation, fifty-one CCS who had previously attended the LTFU clinic took part in a single semi-structured interview to seek their views on information they had received regarding late adverse effects (LAEs) of treatment, the purpose of LTFU and the provision of health promotion information. Although most (93\%) CCS were satisfied with the information received about LAEs, 37\% desired further details. Over half (59\%) believed the purpose of LTFU was to screen for LAEs, while $31 \%$ felt that it was to check for relapse. No survivor reported health promotion to be an aim of LTFU; only $14 \%$ of CCS expected to receive healthy lifestyle advice and fewer than $10 \%$ wanted dietary and physical activity advice. Most (88\%) CCS felt that their hospital-based healthcare professional was best placed to give healthy lifestyle advice but there was no consensus about the optimum timing for health promotion. CCS varied in their knowledge, needs and wishes regarding LTFU care. The results of this evaluation strongly indicate that the profile of health promotion needs to be raised within our service and identifies issues which may be pertinent to similar services. Further research is needed to understand the views of CCS regarding health promotion and lifestyle behaviours, with the aim of tailoring and improving the delivery of effective health education to CCS. 


\section{Introduction}

Advances in the treatment of childhood malignancy have increased survival rates dramatically, reaching 75-80\% in developed countries.[1-3] Consequently, it has been estimated that one in 715 young adults is a childhood cancer survivor (CCS).[4] This rapidly growing population of CCS has stimulated interest in the long-term health consequences of treatments for childhood cancer. There is now well-documented evidence that CCS are vulnerable to several adverse health problems, many of which may not appear until several years or even decades after the end of treatment. Approximately $60 \%$ of long-term survivors will have at least one chronic health condition, $30 \%$ will have at least two and $30 \%$ will experience a severe or life-threatening complication.[5-7] These late adverse effects (LAEs) of treatment may affect a range of organs and systems including cardiovascular, pulmonary, endocrine, reproductive, renal, neurocognitive, auditory, and others.[8,9] In recognition of the potential for treatment-related morbidity, long-term follow-up (LTFU) of CCS aims to facilitate early detection of, or ideally prevent, LAEs of treatment.[10]

In addition, there is considerable emerging interest in how the quality of life of CCS can be improved and health promotion and healthy lifestyle advice are seen as an important part of this aim. Establishing a healthy lifestyle is important for any adolescent or young adult and may be considered even more crucial for those who have experienced cancer and received cytotoxic treatment since their vulnerability to treatment-related adverse health outcomes in later life may be exacerbated by poor health behaviours. In addition, behaviours developed in adolescence are known to track into later life.[10] Although reviews have concluded that smoking and drinking in CCS are at levels similar to or lower than that of comparison groups, these outcomes are nevertheless stated to be at levels that still warrant action.[11-13] Moreover, CCS consistently fail to meet guidelines for physical activity and diet.[11,12,14] 
Modification of health behaviours at a young age may present both healthcare professionals and survivors with a strategy to reduce the risk of LAEs in adulthood.

However, delivering effective health promotion to young people can be particularly challenging in a phase of life characterised by experimentation, risk-taking and increasing independence.[15] Furthermore, research has shown that a large proportion of CCS are not well informed about their previous disease and potential late toxicities of treatment. [16-18] Therefore, health promotion strategies for CCS must also take into account the unique educational needs associated with surviving cancer. [19]

It is widely agreed that providing patients with information about self-management and health promotion should be considered a vital and integral part of LTFU.[13] The transition of CCS from paediatric to adult-centred health services is increasingly recognised as an important stage of LTFU and health education is considered a key component of this process.[20-22] Aftercure, a resource developed specifically for adolescent and young adult CCS, is widely used in LTFU clinics throughout the U.K. [22] Aftercure explains the need for LTFU and provides information on LAEs, lifestyle and other survivorship issues. Several LTFU clinical practice guidelines give specific recommendations for the surveillance of LAEs but provide less guidance for the promotion of healthy lifestyles. [23]

Although there is much research evidence highlighting the need for health promotion in CCS, [11-14,] there is a lack of literature exploring the views of CCS on health promotion, and more specifically the perspectives of adolescent and young adult CCS. Therefore, this service evaluation of a LTFU clinic aimed to ascertain teenage and young adult survivors' experiences and views on the provision of information regarding future health risks, disease prevention and health promotion, including how, when and by whom it might be provided. 


\section{Materials and methods}

\section{LTFU Service}

This service evaluation was of a LTFU clinic at a regional paediatric / adolescent oncology unit serving the North of England's population. The unit is one of the largest in the U.K. and treats all cases of childhood cancer and leukaemia in the region. CCS enter LTFU approximately 5 years after treatment and are most commonly seen annually. The practice of the LTFU clinic is to inform survivors of their future health risks and of the impact of adverse health behaviours by provision of, and verbal discussions around, individualised treatment summaries / care plans, as well as more generic advice specifically written for CCS such as Aftercure. [22]

\section{Survivors}

Participants were survivors of childhood and adolescent cancer currently in LTFU care at the regional unit. Inclusion criteria were that survivors had been treated for any childhood or adolescent malignancy, were at least 14 years old at the time of interview; had completed treatment at least 4 years ago; had previously attended the LTFU clinic and were English speaking.

\section{Procedure}

Recruitment for the survey employed stratified purposive sampling to ensure varied population with regards to initial diagnoses and treatments received. This was to enable access to a broad range of experiences and views. Patient lists of forthcoming LTFU clinics were reviewed by a consultant paediatric / adolescent oncologist and LTFU nurse specialist and checked against the inclusion criteria. Survivors identified as being eligible were approached by the LTFU Nurse Specialist whilst attending their routine appointment at the LTFU outpatient clinic and were invited to take part in the service evaluation. They were 
informed that participation would involve a one-off interview with a medical student (JM), either before or after their clinic appointment with their consultant paediatric / adolescent oncologist. Survivors aged 14-18 years old were given the choice to be accompanied by their parents. Prior to interview, informed verbal consent was obtained from all survivors, and from the parents of survivors aged $<18$ years old. Participants were informed that there were no right or wrong answers, their participation in, or withdrawal from, the interview would not affect their care and that the medical student was not part of their LTFU care team. The interview lasted for as long as the participant wished to speak. As this was a service evaluation aiming to judge current care provided by an LTFU clinic, with the intention of informing future practice, no patient-identifiable data was collected and ethical approval was not required.

\section{Data collection}

Data collection was conducted over a six month period. Data was collected via interviews, which took place in a private consulting room in the clinic. Interviews were guided by a semi-structured schedule consisting of open questions that allowed survivors to talk freely. To gain survivors' specific views on lifestyle topics e.g. which topic did they most want to receive information on whilst at LTFU, survivors were asked to indicate their answer from a list of topics. The interview schedule was initially piloted in interviews with five CCS attending LTFU to ensure the questions were clear to survivors whilst also providing data which was used in the service evaluation. After minor amendments, the interview schedule was then employed in subsequent interviews with CCS attending LTFU. A summary of the interview schedule is provided in Table 1. Survivors were also given the opportunity to discuss other issues important to them. The interviewer took detailed field notes of the survivors' responses during the interview.

\section{Data analysis}


Data were analysed using content analysis.[24] Content analysis is a systematic and objective process which enables the reduction of data by establishing categories and recording the frequency at which they are present in the data.[25,26] Content analysis enables replicable and valid inferences from data to their context to provide researchers with new insights or greater understanding of the topic and can also inform practical actions.[27] This partially quantitative method involved examining the text from the interviews and agreeing on the units of analysis for the categories which in the present service evaluation were words, phrases and sentences in the data. A coding frame was developed to enable organisation of the data and to record the number of occurrences of each category within the text. [28] This allowed the data to be presented numerically using frequencies and percentages and ensured that coding was consistent The coding frame and the subsequent analysis were discussed and agreed with an experienced qualitative researcher to aid the credibility of the findings. 


\section{Results}

\section{Participants' characteristics}

Prior to clinic, 55 participants were identified as being eligible and of these, 51 (93\%) attended their appointment. All 51 survivors approached at clinic agreed to take part in a oneto-one interview with interviews lasting between 20-40 minutes. Twenty-five males and 26 females took part, with an age range of 14-33 years old at time of interview (Table 2). Of the eight (16\%) aged between 14-17 years, six (75\%) chose to be interviewed without their parents present. Both haematological and solid tumour diagnostic groups were well represented with 22 and 29 survivors respectively. The solid tumour group included both central nervous system $(n=6)$ and extra-cranial $(n=23)$ tumours. Age at diagnosis ranged between 2 and 22 years old, with time since the end of treatment ranging from 5 to 19 years.

\section{Survivors' views on information provided by clinics}

Almost all of the survivors $(n=47,93 \%)$ were satisfied with the information they had received about LAEs from the consultant paediatric / adolescent oncologist and specialist nurse during LTFU. Upon further questioning three quarters of the survivors $(n=38,75 \%)$ stated the potential LAEs of treatment were well explained. However, seven survivors (14\%) could not remember this information being given and six (12\%) felt it had not been explained well. The reasons given for this were that the information was confusing or the timing of the discussion had not been appropriate for them in that they were too young or conversely for others, they felt they should have received this information sooner.

Furthermore, 10 (20\%) survivors expressed concerns about future side-effects of treatment and over a third $(\mathrm{n}=19,37 \%)$ commented that, if available, they would like further information about possible side effects that might result from the treatment they had actually received. 


\section{Survivors' views on the purpose of LTFU}

Survivors had varying beliefs concerning the purpose of attending LTFU appointments. Whilst over half of the survivors $(n=30,59 \%)$ thought clinics were intended to screen for LAEs, almost a third $(n=16,31 \%)$ believed their attendance at the clinic was to check if their original malignancy had returned. Health promotion was not reported by any survivor to be a purpose of LTFU and when prompted, only seven (14\%) agreed that they would expect to receive lifestyle advice whilst attending LTFU.

All survivors stated that they expected to see their consultant paediatric / adolescent oncologist while at clinic, and all reported that the appointment length was satisfactory and that they felt they had ample opportunity to ask questions.

\section{Survivors' views on lifestyle advice and health promotion}

Over half of the survivors $(n=30,59 \%)$ believed lifestyle advice was more important for cancer survivors than it was for the general population, with the remainder stating that it was of equal importance. There was a general perception amongst most of the survivors (46/51, $90 \%$ ) that healthy lifestyle information was "general knowledge" and that they "know most of it already". Common sources of lifestyle information and advice were reported to be the internet (49\%), school (12\%), magazines and TV (12\%), friends and family (10\%) or spoken/written information from hospital staff (10\%).

Survivors were asked what health information they would like to receive at LTFU appointments (Fig. 1). The most common topics survivors wished to receive information about were checking lumps and bumps $(\mathrm{n}=12,24 \%)$ and reducing stress $(\mathrm{n}=8,15 \%)$. Fewer survivors stated they would like to receive information on checking moles and skin changes $(n=5,10 \%)$, physical activity $(n=4,8 \%)$ and sun protection $(n=4,8 \%)$. Nobody expressed a wish to receive information about safe sex and contraception. Eleven (21\%) survivors stated 
there were no lifestyle topics they would like to receive advice and information at the LTFU clinic.

Survivors were then asked which single topic from a list was most important to them (Fig. 1). Checking lumps and bumps, physical activity and exercise, and dietary advice were of equal importance (all $n=10,20 \%$ ) as was reducing stress and smoking cessation (both $n=5,10 \%$ ). Survivors were also asked which topics they felt they knew the least about. The most commonly reported topics were dental hygiene, $(n=8,16 \%)$ reducing stress and checking lumps and bumps ( $\mathrm{n}=6,12 \%)$. However, 18 survivors $(35 \%)$ did not report there to be any topics they knew the least about.

\section{Survivors' views on who should discuss health promotion and when}

The majority of survivors $(n=46,90 \%)$ said they had discussed lifestyle advice at some stage during treatment or LTFU. Approximately half of survivors $(n=28,55 \%)$ believed that the initiative to begin a health promotion conversation during LTFU should lie with the consultant paediatric / adolescent oncologist. The remainder believed that consultants should be prepared to discuss these issues if the survivor wished but that these conversations should not be mandatory. Most survivors $(n=45,88 \%)$ felt a separate appointment for lifestyle advice and discussion would not be necessary.

Most survivors ( $\mathrm{n}=45,88 \%$ ) commented that if lifestyle advice were to be discussed, a hospital-based healthcare professional such as their consultant paediatric / adolescent oncologist or specialist nurse would be the best person to provide advice, whilst the remainder felt that their general practitioner (GP) would be the best person to ask. There was no agreement concerning when this information should be given to patients. Forty-five (88\%) survivors agreed it is important to receive further information and that they would like to 
know the full facts and consequences of their treatment, but also that they did not want to be continually reminded when more immediately important topics might be on their mind.

\section{Survivors' views who should provide LTFU and where}

All survivors agreed that the paediatric / adolescent clinic was an appropriate location for their LTFU. No survivors reported concerns about their follow-up in a paediatric outpatient clinic and none wished to transfer their care to an adult clinician. Only 10 (20\%) survivors stated they would consider transferring to a specialist young adult unit and the remainder felt transferring to these services was unnecessary. Their reasons for not wanting to make the transition to an adult clinician included the level of trust they had in the current staff $(n=30$, $59 \%)$, not wanting to be cared for by a new doctor/team $(n=18,36 \%)$, feeling they would be discharged soon anyway $(n=2,4 \%)$, and believing the adult clinician wouldn't know enough about their condition $(n=1,2 \%)$.

If survivors had any problems regarding their general health or lifestyle choices, over half $(n=31,61 \%)$ said they would phone their hospital team whilst $20(39 \%)$ said they would contact their GP first. Travel time and convenience was an important reason survivors preferred to see their GP. Following a brief explanation of the role of a key worker in LTFU care (a professional who acts as a point of contact throughout a patients LTFU journey, providing information and support), 33 (65\%) commented that they would use a key worker as their first contact for any health issues. 


\section{Discussion}

This service evaluation of a LTFU clinic aimed to survey CCS about their experiences and views of health promotion and information provision including their opinions about when and how the potential LAEs of their previous treatment should be discussed, whether lifestyle advice should be discussed, possible ways of achieving this and their views about follow-up. The service evaluation found that although the majority of survivors perceived the risks of treatment had been explained well during LTFU, there was still demand for further information regarding potential health risks. Indeed, despite initially reporting being satisfied with the information they had received on LAEs, some survivors went on to state otherwise in that they felt LAEs were either poorly explained or they had no recollection of them being explained. The findings also suggested that although many survivors are aware that LTFU aims to monitor their health for the development of LAEs, a large proportion still perceive the purpose of the LTFU clinic to be surveillance for their original disease. Despite this focus on the monitoring of health, no survivors reported health promotion to be a key component of LTFU with very few stating they would expect to receive lifestyle advice while at clinic. The survivors in this service evaluation, most of whom were young adults, also reported a desire to stay within the paediatric and adolescent clinic as opposed to transferring to adult services.

Recent research has highlighted that survivors may want more information about potential LAEs. Similar to this patient survey, Knijnberg (2010) reported that although $96 \%$ of CCS responded that the information received at LTFU was sufficient, $29 \%$ went on to state that they had unanswered questions.[28] Although the survivor's knowledge of their potential risk of LAEs was not evaluated in the current service evaluation, previous research has consistently indicated that the knowledge survivors possess about LAEs, as well as their original disease, is often lacking.[16, 29, 30] For CCS to be able to take responsibility for 
their own health, it is essential that they are aware of their potential health risks, as well as how these risks may be attenuated.

Previous research has suggested that survivors differ in their expectations of LTFU, with some CCS expecting a clinical approach and others anticipating more supportive care. [31] In the current service evaluation, clinical reasons were stated as being the main purpose for LTFU, namely monitoring for LAEs (59\%) and to a lesser extent monitoring for the recurrence of the cancer (31\%). Although screening for the original disease may continue to be an objective of prolonged follow-up in some cases (e.g. brain tumours), for most survivors it is important to clarify that the main purpose of LTFU is to inform CCS about, and perform surveillance for, LAEs of treatment. A British study similarly reported that late effects and current health were the two main topics survivors wished to discuss during LTFU. However, unlike the present survey, over $50 \%$ of young survivors also wanted to discuss health behaviours at their clinic appointment. [32] The discrepancies between the findings of this study and ours may be explained by the differences in samples, with Absolom's participants being both older at diagnosis (young adult cancer survivors as opposed to CCS) and at current age (mean 37.9 years old).

Although very few CCS stated that they initially expected to receive lifestyle advice at LTFU, several survivors proceeded to indicate areas in which they would like to receive information, in particular, checking lumps/bumps and reducing stress. However, few survivors expressed a desire for information regarding key lifestyle behaviours such as smoking, diet and physical activity, with almost a quarter stating there were no lifestyle topics they would like to receive advice on. Although this service evaluation did not collect information on the levels of these health behaviours in this sample, diet and physical activity are consistently identified as being areas of concern within childhood cancer survivors which could have important implications for the development of a number of LAEs involving 
cardiovascular and metabolic health.[11,12,14] Previous studies have also reported higher interest from CCS in receiving lifestyle advice with suggestions that this may often be an unmet need during LTFU appointments.[16, 32] However, these data were collected via questionnaires as opposed to interview which may have influenced the survivors' responses. For instance, in this survey no survivor gave responses regarding safe sex and contraception advice suggesting that they may have been embarrassed to disclose this face-to-face as opposed to doing so in an anonymous questionnaire. To reduce the potential for social desirability bias, the tendency for participants to give favourable responses as opposed to revealing their true feelings, CCS were informed that the medical student was independent to their LTFU care team. The fact that the medical student was of a similar age to many of the participants, may have aided rapport. Informing patients that participation is optional and they can withdraw at any point can also aid honesty in their answers. [33]

Although the contemporary focus of LTFU is to empower survivors, over half of the survivors in this service evaluation felt that discussion of health promotion topics should be initiated by their consultant, as opposed to themselves during their consultation, even though the large majority of survivors included in this survey were over 19 years of age (84\%). In addition, no survivors expressed an interest in their care being transferred to an adult oncologist. Although this reluctance to transfer LTFU care has been observed previously, there is increased awareness amongst healthcare professionals of the potential benefits of age-appropriate adult based care and recognition of the importance of making survivors more aware of these advantages.

This service evaluation also confirmed previous findings that CCS are in favour of LTFU care within the existing consultant-led model. However due to the increasing number of survivors, many of whom are suffering from chronic health conditions, there is an important need to develop alternative services which will provide the best medical care to meet each 
survivor's needs. This survey showed that after explanation of the role of the key worker most survivors commented they would use this person as their first contact for health issues.

A survey of adult cancer survivors aged 18-45 demonstrated that they preferred hospitalbased care rather than GP follow-up.[32] This service evaluation in a younger age group suggests that this is also true for CCS. However, it is pertinent to reflect on the well-known strengths of primary care in providing chronic health condition management, health promotion and holistic care.

Health promotion and lifestyle advice is now generally considered to be a vital part of medical care of CCS. Recent research is providing welcome evidence that adherence to a healthy lifestyle, particularly physical activity and diet, may reduce the risk of developing cardiovascular disease, diabetes and metabolic syndrome in CCS. [34-36] In recent years, many countries have developed guidelines to help clinicians and survivors detect LAEs in a LTFU setting but as yet, these guidelines contain very little guidance on health promotion. At present, 'health links' from the Children's Oncology Group in the United States provides the most detailed guidance on health-related behaviours for CCS. [37] Forthcoming guidelines from the PanCareSurFup (PanCare Childhood and Adolescent Cancer Survivor Care and Follow-Up Studies) consortium will aim to provide evidence-based recommendations for health promotion. However, no guidelines currently give recommendations for how best to communicate this information to CCS to aid sustainable adoption of positive health behaviours by these patients.

This service evaluation has some limitations. As the aim was to collect views on the provision of information at the LTFU clinic, clinic non-attenders were not approached. It was deemed inappropriate to contact non-attenders as they may have had personal reasons for not coming to clinic and it was recognised that an unsolicited approach might carry a risk of 
causing uncertainty or even psychological stress. Although all participants had attended the LTFU clinic more than once, information on the number of visits was not collected. We are unable to know when information on LAEs was given to individual survivors, therefore, we cannot fully interpret their views on when this information should be given. However, it is indicated that preferences may be personal to each survivor and that it may be beneficial for the risk of LAEs to be revisited with the survivor at several time-points.

The 51 survivors surveyed had a wide range of diagnoses, ages at evaluation and durations of follow-up since treatment completion. The treatments they had received encompassed all modalities including chemotherapy, radiotherapy, surgery and haemopoietic stem cell transplantation. This broad sample and the fact that nobody declined to participate in the survey, suggests that participants could be considered a good representation of the patient group the evaluation was targeting. Purposive sampling, a common practice in qualitative research, selects participants from a predefined group who can provide insight into the research question. Using stratification on key variables ensures diversity within this sample to ensure that a range of perspectives are accessed. [38] However, to ensure this diversity, purposive sampling requires an element of judgement on the part of the researcher.

Although survivors' responses were recorded in writing, care was taken to clarify any ambiguous statements and to note the exact phrases used by participants. Handwritten notes do limit the ability to record data verbatim and may be subject to an element of selectivity but may also put at ease those participants who would feel apprehensive of being audio-recorded. This method of data collection requires the interviewer to listen attentively to the participant. [39] Although, audio-recordings provide a full account of what is said, transcription of the data is extremely time-consuming and as this was a service evaluation, resources were limited. However, it is acknowledged that in research, transcription is preferred as it also enables a more in depth method of analysis such as thematic as opposed to content analysis. 
By delivering a service evaluation via interview there was ample time for survivors to use their own words and expand on their answers. In turn, the interviewer could use follow-up questions to obtain more detailed information and identify any contradictions in the responses, clarify questions for survivors if needed, and check that the response noted was deemed accurate by the participant. Using these tactics, as well as guidance from those experienced in qualitative research, aids the 'trustworthiness' of the data. [33] This meant a large amount of information was gathered and survivors' ideas, concerns and expectations could be thoroughly explored. However, it is important to highlight that the findings of qualitative research cannot be stated to be generalisable but rather, as Guba (1981) suggests, may be considered as transferable to similar contexts. [40] Therefore, health professionals within similar LTFU services may feel that our findings may be relevant to their own clinics.

It is important that LTFU addresses the needs of CCS and in most cases lifestyle advice forms part of these needs. Even if it is not obvious that the survivor desires lifestyle advice at the LTFU clinic appointment, it may be worth informing them that there are resources available to survivors. It is worth noting that non-attenders may have even greater knowledge gaps and hence information needs including health promotion.

In conclusion the findings of this service evaluation highlight the importance of finding out what survivors want in order to help clinicians deliver effective survivor-centred care. The survey also demonstrates that survivors vary in their knowledge, needs and wishes in regards to LTFU. These results illustrate the need to raise the profile of health promotion in LTFU. The service evaluation has encouraged a greater focus on health education and promotion practice in our LTFU clinic. However, it is important that efforts to improve clinical practice are informed by a deeper understanding of the views, practices and needs of CCS regarding health behaviours. Such knowledge will enable LTFU services to deliver health education more effectively and to tailor its content more precisely according to survivors' needs, 
offering a possible strategy to improve their long-term outcomes by reducing potentially avoidable morbidity and mortality.

\section{Acknowledgments}

J Mayes was supported by the Newcastle University scholarship fund. MC Brown was

supported by the Children's Cancer Fund. We are grateful to the staff and patients at the Great North Children's Hospital Oncology Unit who helped with this project.

\section{Declaration of Interest}

The authors report no conflicts of interest 


\section{References}

1. Magnani C, Pastore G, Coebergh JW, Viscomi S, Spix C, and Steliarova-Foucher E. Trends in survival after childhood cancer in Europe, 1978-1997: Report from the Automated Childhood Cancer Information System project (ACCIS). Eur J Cancer. 2006; 42:1981-2005.

2. Ellison LF, Pogany L, and Mery LS. Childhood and adolescent cancer survival: a period analysis of data from the Canadian Cancer Registry. Eur J Cancer. 2007; 43:19671975.

3. Gatta G, Botta L, Rossi S, Aareleid T, Bielska-Lasota M, Clavel J, Dimitrova N, Jakab Z, Kaatsch P, Lacour B, Mallone S, Marcos-Gragera R, Minicozzi P, Sánchez-Pérez, MJ, Sant M, Santaquilani M, Stiller C, Tavilla A, Trama A, Visser O, and Peris-Bonet R. Childhood cancer survival in Europe 1999-2007: results of EUROCARE-5 a populationbased study. Lancet Oncol. 2014; 15:35-47.

4. Campbell J, Wallace WHB, Bhatti LA, Stockton DL, Rapson T, and Brewster DH. Childhood cancer in Scotland: trends in incidence, mortality and survival 1975-1999. 2004. Edinburgh: NHS Scotland Information and Statistics Division.

5. Oeffinger KC, Mertens AC, Sklar CA, Kawashima T, Hudson MM, Meadows AT, Friedman DL, Marina N, Hobbie W, Kadan-Lottick NS, Schwartz CL, Leisenring W, and Robison LL. Chronic health conditions in adult survivors of childhood cancer. NEJM. 2006; $355: 1572-82$.

6. Stevens MC, Mahler H, and Parkes S. The health status of adult survivors of cancer in childhood. Eur J Cancer. 1998; 34:694-8.

7. Curry HL, Parkes SE, Powell JE, and Mann JR. Caring for survivors of childhood cancers: the size of the problem. Eur J Cancer. 2006; 42:501-508. 
8. Hudson MM, Ness KK, Gurney JG, Mulrooney DA, Chemaitilly W, Krull KR, Green DM, Armstrong GT, Nottage KA, Jones KE, Sklar CA, Srivastava DK, and Robison LL. Clinical Ascertainment of Health Outcomes among Adults Treated for Childhood Cancer: A Report from the St. Jude Lifetime Cohort Study. JAMA. 2013; 309:2371-2381.

9. Robison LL, and Hudson MM. Survivors of childhood and adolescent cancer: lifelong risks and responsibilities. Nat Rev Cancer. 2014; 14:61-70.

10. Edgar AB, Morris EM, Kelnar CJ, and Wallace WH. Long-term follow-up of survivors of childhood cancer. Endocr Dev. 2009; 15:159-80

11. Rabin, C. Review of health behaviors and their correlates among young adult cancer survivors. J Behav Med. 2011; 34:41-52.

12. Nathan PC, Ford JS, Henderson TO, Hudson MM, Emmons KM, Casillas JN, Lown E A, Ness KK, and Oeffinger KC. Health behaviors, medical care, and interventions to promote healthy living in the Childhood Cancer Survivor Study Cohort. J Clin Oncol. 2009; 27:2363-2373.

13. Clarke SA, and Eiser C. Health behaviours in childhood cancer survivors: a systematic review. Eur J Cancer. 2007; 43:1373-84.

14. Zhang F, Saltzman E, Must A, and Parsons S. Do Childhood Cancer Survivors Meet the Diet and Physical Activity Guidelines? A Review of Guidelines and Literature. Int J Child Health Nutr. 2012; 1:44-58.

15. Viner R, \& Macfarlane A. Health promotion. BMJ. 2005; 330:527-529. 
16. Kadan-Lottick NS, Robison LL, Gurney JG, Neglia JP, Yasui Y, Hayashi R, Hudson M, Greenberg M, Mertens AC. Childhood cancer survivors' knowledge about their past diagnosis and treatment: Childhood Cancer Survivor Study. JAMA. 2002; 287:1832-9.

17. Ford JS, Chou JF, Sklar CA. Attendance at a survivorship clinic: impact on knowledge and psychosocial adjustment. J Cancer Surviv. 2013; 7:535-43.

18. Kunin-Batson A, Steele J, Mertens A, Neglia JP. A randomized controlled pilot trial of a Web-based resource to improve cancer knowledge in adolescent and young adult survivors of childhood cancer. Psychooncology. 2015. doi: 10.1002/pon.3956.

19. Hudson MM, and Findlay S. Health-risk behaviors and health promotion in adolescent and young adult cancer survivors. Cancer. 2006; 107:1695-701.

20. Henderson TO, Friedman DL, and Meadows AT. Childhood cancer survivors: transition to adult-focused risk-based care. Pediatrics. 2010; 126:129-36.

21. Freyer DR. Transition of care for young adult survivors of childhood and adolescent cancer: rationale and approaches. J Clin Oncol. 2010; 28:4810-4818.

22. Children's Cancer and Leukaemia Group. Aftercure: a guide for teenage \& young adult survivors of childhood cancer. 2016. Available at: http://www.aftercure.org/ [Accessed 25 Feb. 2016]

23. Brown MC, Levitt GA, Frey E, Bárdi E, Haupt R, Hjorth L, Kremer L, Kuehni CE, Lettner C, Mulder RL, Michel G, Skinner R; on behalf of the PanCareSurFup Consortium. The views of European clinicians on guidelines for long-term follow-up of childhood cancer survivors. Pediatr Blood Cancer. 2015; 62:322-328. 
24. Joffe H, and Yardley L. Content and thematic analysis. In: Marks D, Yardley L, ed. Research Methods in Clinical and Health Psychology. London: SAGE; 2004:56-68.

25. Waltz C, Strickland O, and Lenz E. Measurement in Nursing Research. New York: Springer Publishing Company. 2010.

26. Krippendorff K. Content Analysis: An Introduction to its Methodology. Los Angeles: SAGE. 2013.

27. Berg BL. Qualitative Research Methods for the Social Sciences. Boston: Allyn and Bacon. 2001.

28. Knijnenburg SL, Kremer LC, Van Den Bos C, Braam KI, and Jaspers MW. Health information needs of childhood cancer survivors and their family. Pediatr Blood Cancer. 2010; 54:123-7.

29. Eiser C, Levitt G, Leiper A, Havermans T, and Donovan C. Clinic audit for long-term survivors of childhood cancer. Arch Dis Child. 1996; 75:405-409.

30. Absolom K, Greenfield D, Ross R, Horne B, Davies H, Glaser A, Simpson A, Waite H, and Eiser C. Predictors of clinic satisfaction among adult survivors of childhood cancer. Eur J Cancer. 2006; 42:1421-7.

31. Michel G, Greenfield DM, Absolom K, Ross RJ, Davies H, and Eiser C. Follow-up care after childhood cancer: survivors' expectations and preferences for care. Eur J Cancer. $2009 ; 45: 1616-23$

32. Absolom K, Eiser C, Michel G, Walters SJ, Hancock BW, Coleman RE, Snowden JA, and Greenfield DM. Follow-up care for cancer survivors: views of the younger adult. $\mathrm{Br}$ J Cancer. 2009; 101:561-567. 
33. Shenton AK. Strategies for ensuring trustworthiness in qualitative research projects. Education for Information. 2004; 22:63-75

34. Chow EJ, Chen Y, Kremer LC, Breslow NE, Hudson MM, Armstrong GT, Border W L, Feijen EAM, Green DM, Meacham LR, Meeske KA, Mulrooney DA, Ness KK, Oeffinger, K. C., Sklar, C. A., Stovall, M., Van Der Pal, H. J., Weathers, R. E., Robison LL, and Yasui Y. Individual prediction of heart failure among childhood cancer survivors. J Clin Oncol. $2015 ; 33: 394-402$.

35. Jones LW, Liu Q, Armstrong GT, Ness KK, Yasui Y, Devine K, Tonorezos E, Soares-Miranda L, Sklar CA, Douglas PS, Robison LL, and Oeffinger KC. 2014. Exercise and risk of major cardiovascular events in adult survivors of childhood hodgkin lymphoma: a report from the Childhood Cancer Survivor Study. J Clin Oncol. 2014; 32:3643-3650.

36. Smith WA, Li C, Nottage KA, Mulrooney DA, Armstrong GT, Lanctot JQ, Chemaitilly W, Laver JH, Srivastava DK, Robison LL, Hudson MM, and Ness KK. Lifestyle and metabolic syndrome in adult survivors of childhood cancer: A report from the St. Jude Lifetime Cohort Study. Cancer. 2014; 120:2742-2750.

37. Children's Oncology Group. Long-term follow-up guidelines for survivors of childhood, adolescent, and young adult cancers. Version 4.0. 2013. http://www.survivorshipguidelines.org.

38. Braun, V. \& Clarke, V. Successful Qualitative Research: A Practical Guide for Beginners. London: Sage. 2013.

39. Sim J \& Wright C. Research in Health Care: Concept, Designs and Methods. Cheltenham: Nelson Thornes LTD. 2000. 
40. Guba, E.G. Criteria for assessing the trustworthiness of naturalistic inquiries. ECTJ. 1981; 29: 75-81.

\section{Legend}

Table 1. Example topics and questions from the interview schedule

Table 2. Participants' characteristics

Figure 1. Survivors' views on lifestyle advice topics 
Table 1. Example topics and questions from the interview schedule

\section{Survivor's views on the information provided by clinics concerning long-term side-effects of treatment: \\ Do you remember the risks of treatment being explained to you? If so, by whom and when? \\ Do you think enough information was given about the risks? Did you understand it at the time?}

\section{Survivor's views on expectations of LTFU:}

What do you think the purpose of long-term follow-up is?

What do you expect to talk about when attending long-term follow-up?

\section{Survivor's views on lifestyle advice and health promotion:}

Are there any lifestyle topics that would be helpful for you to know more about?

Which lifestyle topics would like to receive advice about in long-term follow-up clinics?

Which lifestyle topics do you feel are the most important?

For these questions, survivors were shown a prompt card with a list of lifestyle topics (shown in

Figure 1) from which they were asked to indicate their answer.

\section{Survivor's views on transition to adult care:}

Where would you like your long-term follow-up appointments to take place?

Does it matter to you that your appointments are in the paediatric clinic?

\section{Survivor's views on alternative methods of follow-up:}

Who would you contact if you had any questions regarding lifestyle choices or your health?

Do you know who your key worker is? Would you consider contacting them? 
Table 2. Participants' characteristics

\begin{tabular}{|c|l|}
\hline $\begin{array}{c}\text { Characteristic } \\
\text { Gender }\end{array}$ & Survivors ( $\mathrm{n}=51)$ \\
Female & 26 \\
Male & 25 \\
\hline $\begin{array}{c}\text { Diagnosis } \\
\text { Leukaemia }\end{array}$ & 12 \\
Central nervous system tumour & 6 \\
Extracranial tumour & 10 \\
\hline $\begin{array}{c}\text { Age at diagnosis (years), } \\
\text { Median (range) }\end{array}$ & $11.3(2-22)$ \\
\hline $\begin{array}{c}\text { Age at interview (years), } \\
\text { Median (range) }\end{array}$ & $21.8(14-33)$ \\
\hline $\begin{array}{c}\text { Time since treatment (years), } \\
\text { Median (range) }\end{array}$ & 8 \\
\hline
\end{tabular}


Figure 1. Survivors' views on lifestyle advice topics

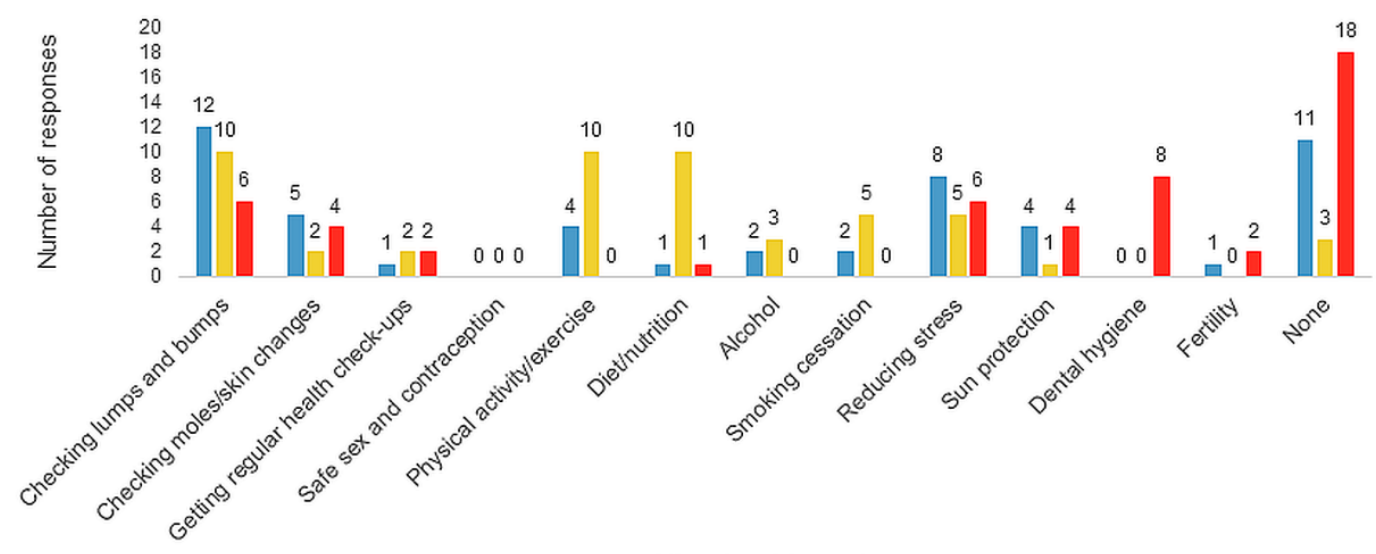

Lifestyle advice topics

घ Topics survivors would like to receive information on in LTFU clinic

$\square$ Topics survivors felt were the most important

- Topics survivors felt they knew the least about 